\title{
A full-disk analysis of pattern of solar oscillations and supergranulation
}

\author{
A. Vecchio \\ ${ }^{1}$ Dipartimento di Fisica, Università della Calabria, 87036 Rende (CS), Italy \\ 2 Istituto Nazionale di Fisica della Materia, Unità di Cosenza, 87036 Rende (CS), Italy \\ e-mail: vecchio@fis.unical.it \\ Received 25 July 2005 / Accepted 8 September 2005 \\ ABSTRACT \\ We present the results obtained by applying the Proper orthogonal Decomposition (POD) technique to analyzing photospheric full disk veloc- \\ ity fields acquired by the Magneto-Optical Filter (MOF) Development group at the Kanzelhöhe solar observatory. Using this technique, we \\ were able to identify and characterize the different dynamical regimes acting in the system. The solar rotation dominates the most energetic \\ POD modes. Using a time series of only $256 \mathrm{~min}$, the POD can identify and isolate the solar 5-min oscillations and the solar supergranulation \\ pattern.
}

Key words. Sun: photosphere - Sun: granulation - Sun: oscillations - methods: data analysis

\section{Introduction}

Photospheric convective flows cover a wide range of spatial and temporal scales, so that simultaneous studies of the different scales are not straightforward. The most important and known patterns are granulation with a typical spatial scale of about $1000 \mathrm{~km}$ and supergranulation with scales around $30000 \mathrm{~km}$. In the literature many studies of the granulation cellular pattern have been performed with different approaches (see e.g. Bray et al. 1984; Spruit et al. 1990). The solar supergranulation is observed in its horizontal flow component either by direct Doppler measurements away from disk center (e.g. Hart 1954, 1956; Leighton et al. 1962; Deubner 1971; Giovannelli 1980; Rimmele \& Schröter 1989; Srikanth et al. 2000; Hathaway et al. 2002) or near disk center by tracking the advection of magnetic elements or smaller scale flows (e.g. Simon 1967; November \& Simon 1988; Simon et al. 1988; Wang \& Zirin 1988; Muller et al. 1992; November 1994; Wang et al. 1996; Zhang et al. 1998; Roudier et al. 1999; De Rosa et al. 2000; Shine et al. 2000; Lisle et al. 2000). The supergranular flow pattern appears as an ensemble of cells, diverging from the center outward and the cell boundaries are characterized by a strong photospheric magnetic field and chromospheric network (Simon \& Leighton 1964). The horizontal velocity is most frequently in the range $300-500 \mathrm{~m} / \mathrm{s}$, and the velocity distribution extends up to $\sim 1 \mathrm{~km} \mathrm{~s}^{-1}$ (Shine et al. 2000), while the vertical velocity of supergranulation attains lower values $(50-100 \mathrm{~m} / \mathrm{s})$ (Küveler 1983). Studying the supergranulation pattern is not easy because observations do not show a clear contrast in white light. Moreover, ground-based observations are complicated by the large mean life time ( $\sim 1$ day) of supergranulation. Thus indirect tracers, such as the magnetic field and cromospheric emissions, are commonly used to identify the flow cells.

On the solar surface, the contribution of solar global oscillations (5 min p-modes) is superimposed on the turbulent evolution of granulation and supergranulation, and the spatial and temporal scales of p-modes are very close to the granular ones (Leighton et al. 1962; Ulrich 1970; Cristensen-Dalsgaard 2002).

In this paper we show how the proper orthogonal decomposition (POD) analysis of line of sight (LOS) photospheric velocity fields is able to distinguish the three different physical phenomena present on the solar surface images withoud any doubt: solar rotation, solar oscillations, and supergranulation. In an astrophysical context, POD has recently been used to analyze the spatio-temporal dynamics of the solar cycle (Carbone et al. 2002; Mininni et al. 2002; Vecchio et al. 2005). In the next section we briefly describe the POD, then present the result of POD applied to LOS velocity fields of the solar photosphere, and finally we discuss the results and their perspectives.

\section{Proper orthogonal decomposition}

The POD procedure is designed to yield a complete set of eigenfunctions that are optimal in energy compared to any other basis (Holmes et al. 1996). Given an ensemble of fields 
- in our case the LOS photospheric velocity fields $u(x, y, t)$ at different times - we introduce the expansion

$u(x, y, t)=\sum_{j=0}^{\infty} a_{j}(t) \Psi_{j}(x, y)$

where the coefficients $a_{j}(t)$ are the temporal modal coefficients, $\Psi_{j}(x, y)$ the POD basis function that is not fixed a priori, and $x, y$ the coordinates on the surface of the Sun. The base given in (1) is built directly from the experimental data set and is obtained by maximizing the averaged projection of $u(x, y, t)$ onto each $\Psi_{j}(x, y)$ constrained to a unitary norm. This leads to a constrained optimization problem that can be cast as a Fredholm integral equation

$\int_{L_{x, y}} \mathrm{~d} x^{\prime} \mathrm{d} y^{\prime}\left\langle u(x, y, t) u^{*}\left(x^{\prime}, y^{\prime}, t\right)\right\rangle \Psi\left(x^{\prime}, y^{\prime}\right)=\lambda \Psi(x, y)$

where $\Psi(x, y)$ is a generic eigenfunction $\Psi_{j}, L_{x, y}$ represents the lengths of spatial integration domain, and the brackets represent averages over all times. The unique optimal basis elements $\Psi_{j}$ are provided by the eigenfunctions of this equation. The POD temporal coefficients are computed by projecting the signal onto the corresponding basis functions. The eigenvalues are a countable set representing the average energy contained in each mode, and they can be ordered such that $\lambda_{j} \geq \lambda_{j+1}$. Finally from the diagonal decomposition of the autocorrelation function, it follows that $\left\langle a_{n}(t) a_{m}(t)\right\rangle=\delta_{n m} \lambda_{n}$; that, is the temporal coefficients for different POD modes are uncorrelated on average and represent the mean energy.

Once the Fredholm equation has been solved and eigenvalues and eigenfunctions have been obtained, a reconstruction of the original field by selectively choosing a finite number $N$ of the most energetic modes is possible. In this way, we form a subspace spanned by the first $N$ eigenfunctions

$u_{N}(x, y, t)=\sum_{j=0}^{N} a_{j}(t) \Psi_{j}(x, y)$.

These eigenfunctions can be used either to build a reduced model of the dynamical behavior of the system or, in an indirect way, to quantify the number of relevant modes for the dynamics.

POD is an "optimal" field expansion, namely a truncated POD expansion as (3) describes typical members of the ensemble better than any other basis of the same truncation order (Holmes et al. 1996). For instance when dealing with a periodic field, the empirical eigenfunctions would just be the Fourier basis (Holmes et al. 1996). Being extracted directly from experiments, POD eigenfunctions $\Psi_{j}(x, y)$ can assume the proper functional shape of the phenomenon, and the associated temporal part $a_{j}(t)$ represents the time evolution of the $j$-th mode associated to that eigenfunction.

It must be realized that POD codes need massive computer resources to solve the Fredholm integral equation, so it is difficult to work with large data sets. The algorithm used in this work follows the snapshot method (Holmes et al. 1996). The allocated memory depends on the temporal points of the data set $T$ and is equal to $T^{2} \cdot n b$, where $n b=4$ in single precision. The calculation can be performed on a personal computer with the suitable calculation resources.

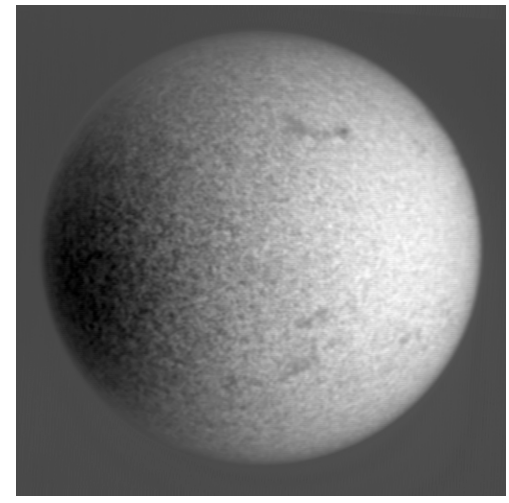

Fig. 1. Example of a snapshot of the data set.

\section{Data analysis}

The line of sight velocity field $u(x, y, t)$ used in this work was obtained from full disk images acquired by P. F. Moretti and the MOF Development group at Kanzelhöhe solar observatory on January 30th 1998 (Moretti et al. 2000). The time series is obtained using a magneto-optical filter centered on sodium D lines $(589.0 \mathrm{~nm}$ and $589.6 \mathrm{~nm})$. An example of a snapshot of the data set is presented in Fig. 1. Detailed information about the measurement, the data reductions, and the calculation of the LOS velocity field can be found in Moretti et al. (2000). The image size is $512 \times 512$ pixels, with a pixel size of about 4 arcsec. The time series spans a time interval $T=257 \mathrm{~min}$, with images being sampled every minute. The POD of the field $u(x, y, t)$, yields a set of 257 eigenfunctions $\psi_{j}(x, y)$ and coefficients $a_{j}(t)$, as well as the sequence of eigenvalues $\lambda_{j}$ $(j=0,1, \ldots, T)$. Most of the energy (99\%) is associated with the first POD mode accounting for the line of sight component of solar rotation. The rest of the energy is decreasingly shared by the following 257 modes, so that for example when excluding the solar rotation contribution, only $38 \%$ of the remaining energy is associated to the second $(j=1)$ POD mode, and the $47 \%$ is contained in the modes $j=1,2$. In Fig. 2 we report the cumulative energy for the first 20 POD mode, defined as

$E_{\text {cum }}(j)=\sum_{k=0}^{j}\left(\lambda_{k} / E_{\text {tot }}\right)$.

In laboratory turbulent flows, PODs attribute almost $90 \%$ of the total energy to the typical large-scale coherent structures confined to $j \leq 2$ (Alfonsi \& Primavera 2002). In our case, $99 \%$ of the energy (excluding the rotation) is contained in the first 75 modes. This indicates the absence of dominating largescale structures and the presence of some turbulent dynamics related to nonlinear interactions among different modes and structures on all scales.

We now turn the attention to the eigenfunctions, namely the spatial pattern found by POD and coefficients describing the temporal behavior of the mode. The POD is a technique developed to study the fluctuations of a turbulent field. If the data set contains the average field, the most energetic POD mode will describe the average part of the field. As already stated, the high energy POD mode, $j=0$, contains the contribution to the solar rotation, wich is the most energetic motion present 


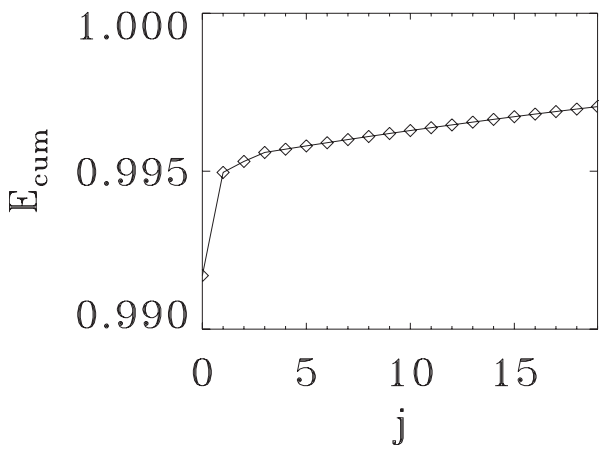

Fig. 2. Cumulative energy of the first 20 POD coefficients as a function of the index $j$.

in the data set, as it is clearly visible in Fig. 3. The temporal behavior of this modes shows the well-known linear variation of the solar rotation due to the relative velocity between the Sun and the observer on the Earth (Moretti et al. 2000). The average value found is about $845 \mathrm{~m} / \mathrm{s}$. Small amplitude 5-min oscillations are superimposed on the dominant rotation trend (Fig. 3). The eigenfunction of the first fluctuating POD mode, $j=1$, presents an evident center-limb modulation. A granular structure is evident in the solar limb region (Fig. 3). The time behavior of $j=1$ mode again shows the linear variation in time with the same slope, in absolute value of the coefficient $a_{0}(t)$ (Fig. 3). Thus we can associate this mode to the persistence of solar supergranulation during the time interval spanned by our data set. As already stated,supergranulation features have characteristic temporal scales larger than the length of the data set.

The coefficients $j=2$ and $j=3$ do not show a regular behaviour and could be due to the turbulent nature of supergranulation. Their power spectra, after smoothing to remove the five-minute oscillations' noise, are characterized by a power law decay (Fig. 5). In the first $j=1,2,3$ POD modes, the contributions of 5-min oscillations are always present and they cannot be completely removed.

Higher $j$ modes describe the solar five-minute oscillations. These modes indicate a predominantly vertical motion with structures localized near the center of the Sun (see the pattern of $\psi_{4}(x, y)$ in Fig. 4). The behavior of temporal coefficient $a_{4}(t)$ (Fig. 4) is predominantly oscillating and, although modulated, is dominated by 5 -min oscillations. In the right hand panel of Fig. 5, the power spectra of the coefficient $a_{4}(t)$ is shown. A peak around a period of $5 \mathrm{~min}$ is clearly visible.

To characterize the spatial scales involved, a projection on the $(r, \theta)$ plane is made for each eigenfunction in order to calculate and analyze the radial wavevector spectra. The projected eigenfunctions are shown in Fig. 6. In this representation, the eigenfunction $\Psi_{0}(x, y)$ shows large structures at solar limb (high $r / R_{\odot}$ ) that is proper to the solar rotation. The supergranular pattern at intermediate solar radius can be observed in the eigenfunction $\Psi_{1}(x, y), \Psi_{2}(x, y), \Psi_{3}(x, y)$. The contribution of p-modes dominates in the eigenfunction $\Psi_{4}(x, y)$ and is larger around the center of the Sun (small $r / R_{\odot}$ ). In Fig. 7 the power spectra $\left|\psi_{j}\left(k_{r}\right)\right|^{2}$ of wavevectors obtained from Fourier transform of the eigenfunctions in the $(r, \theta)$ plane are represented. The eigenfunction $j=0$ contains only the contribution
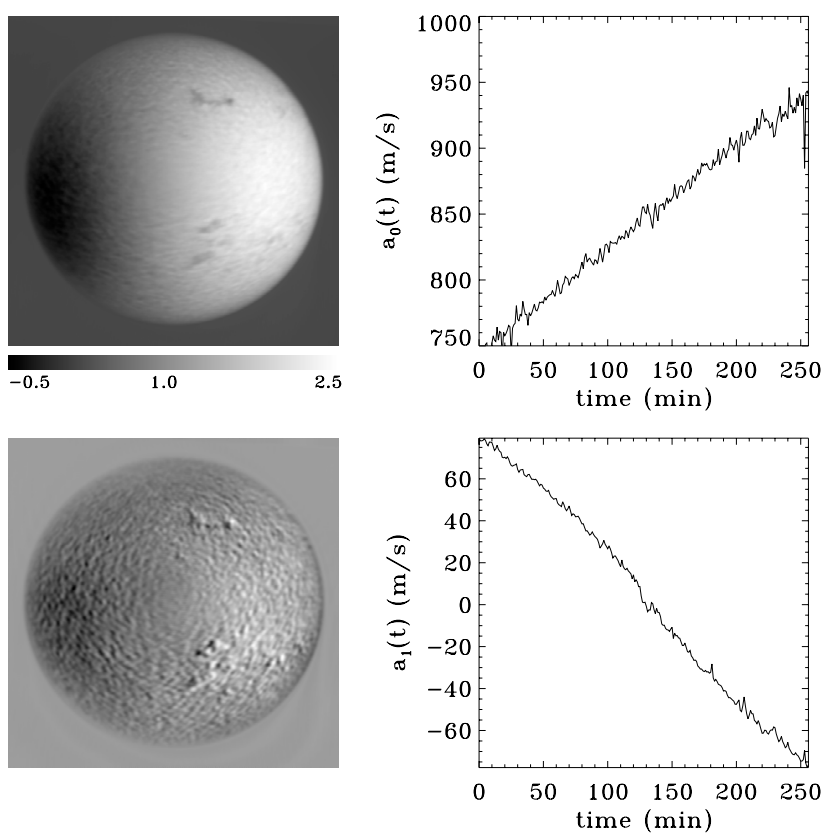

Fig. 3. Left column from top to bottom: the POD eigenfunctions $\Psi_{j}(x, y)$ in the plane $(x, y)$ for the modes $j=0$ and $j=1$. Right column: correponding POD coefficient $a_{j}(t)$.

of solar rotation, and its power spectra is not presented here. For eigenfunctions $j=1, \ldots 4$, we calculated the power spectrum $P\left(k_{r}\right)$ averaged over the $\theta$ coordinate

$P\left(k_{r}\right)=\frac{1}{2 \pi} \sum_{\theta_{i}=0}^{2 \pi} P\left(k_{r}, \theta_{i}\right)$

with

$P\left(k_{r}, \theta\right)=\tilde{v} \tilde{v}^{*}$

and

$\tilde{v}\left(k_{r}, \theta\right)=\int_{\Delta_{r}} v(r, \theta) \exp \left[-i\left(k_{r} r\right)\right] \mathrm{d} r$.

The power spectra are calculated for three different bands of $\Delta r \sim 0.33 R_{\odot}$ moving from the center to the limb. In this way we can quantify the center-limb modulation of the eigenfuntions and correct the different spectra for the projection effect that becomes relevant near the solar limb. To take the projection effect into account, every wavevector in each band is multiplied by the factor $\sqrt{1-\left(\bar{r} / R_{\odot}\right)^{2}}$, where $\bar{r}$ is the average radial distance of the band.

The four power spectra shown in Fig. 7 are broad and characterized by well-defined behaviour. The spectrum $P\left(k_{r}\right)$ for the eigenfunction $j=1$ presents the same behavior for the three bands with enhancement of the power for values of $k$ corresponding to typical supergranular scales around $3.5 \times 10^{4} \mathrm{~km}$. The power is higher for the intermediate band, so that the predominant phenomenon is characterized by horizontal motions. This agrees the interpretation given to this mode describing the persistence of supergranular structures. An increase of power is observed in the high $k$ range, and the spectrum calculated for the band nearest to the center becomes the dominant one. This 

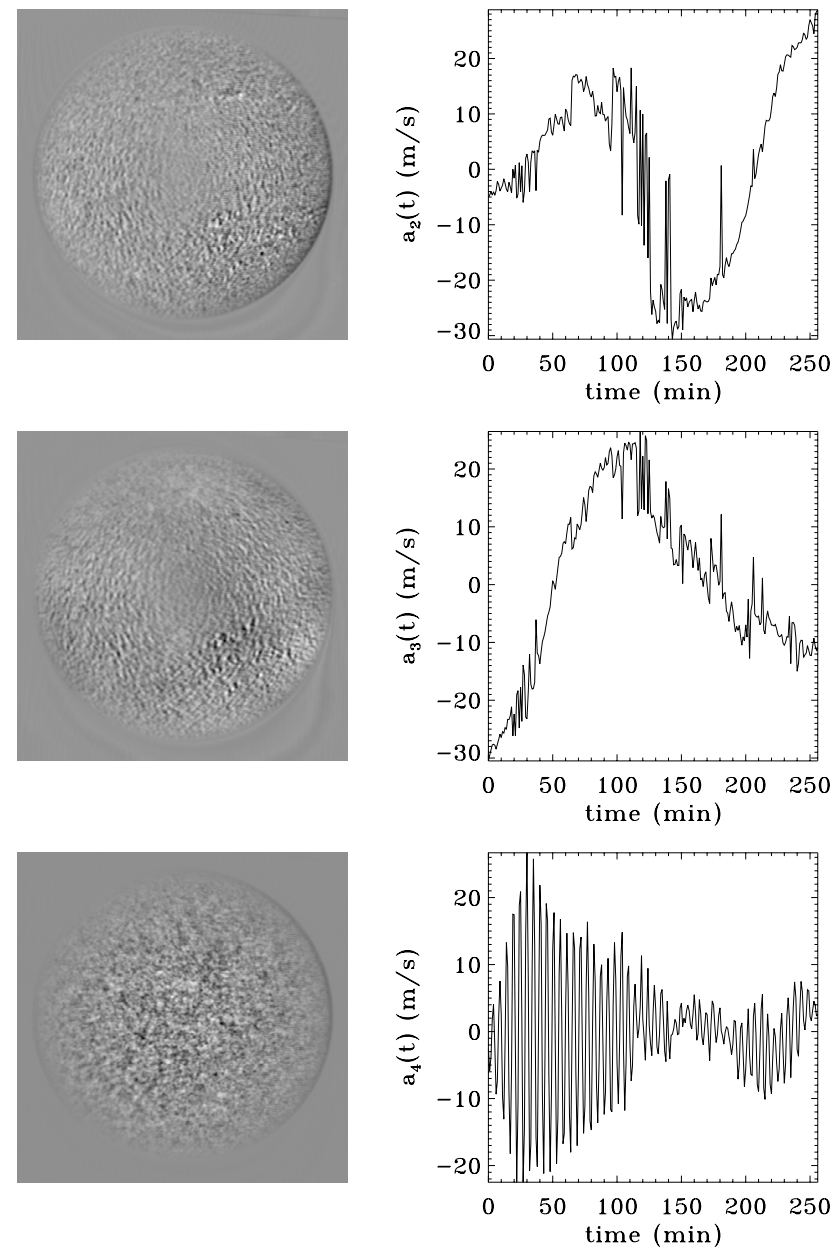

Fig. 4. Left column from top to bottom: the POD eigenfunctions $\Psi_{j}(x, y)$ in the plane $(x, y)$ for the modes $j=2, j=3$, and $j=4$. Right column: corresponding POD coefficient $a_{j}(t)$.
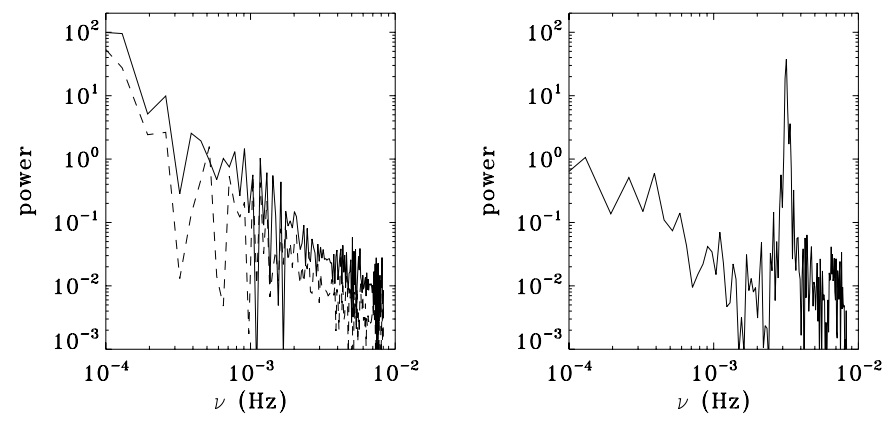

Fig. 5. Left panel: power spectra of the POD coefficients $a_{2}(t)$ and $a_{3}(t)$ (dashed line). Right panel: power spectra of the POD coefficient $a_{4}(t)$.

is due to the contributions of solar oscillations characterized by vertical motions, thus dominant in the center of the Sun. For the eigenfunctions $\psi_{2}(r, \theta)$ and $\psi_{3}(r, \theta)$, the dominant spectra are those calculated in the intermediate bands. The spectra show a very broad peak around $k \simeq 2 \times 10^{-5}$ corresponding to the supergranular scales. The effect of limb darkening is also visible in the spectra. Because of decreasing resolution in the external band, the cellular supergranular structures are not well characterized, and the power is distributed to small $k$. For this reason, for modes $j=1,2,3$, the power spectra calculated in the external band are the lowest and do not show the enhancement for values of $k$ corresponding to the supergranular scales.

The eigenfunction corresponding to the mode $j=4$, dominated by the contribution of p-modes oscillations, presents broad spectra in which no typical scale can be measured. The spectrum for the band in proximity of the solar center is indeed higher than the others, which is evidence of predominant vertical oscillations.

\section{Conclusion}

In this paper we have presented a new technique to investigate both the $p$-modes and the supergranulation pattern by using the longitudinal photospheric velocity field as obtained at the Kanzelhöhe solar observatory. The velocity fields at different times were used as an ensemble of different contributions to gain some insight into the complex spatio-temporal nature of turbulent phenomena. The POD is the best up-to-date technique for capturing the energetic properties of the phenomenon, giving information on the dynamics of the coherent structures and providing an orthogonal empirical basis. Our main results are:

1. Through the POD expansion we found that the first mode $(j=0)$ is associated to the contribution of solar rotation, where the energy is mainly concentrated. The modes $j=1,2,3$ describe the supergranular pattern, and the next less energetic modes $(j \geq 4)$ describe the "5-min" oscillations. The pattern recovered from the first two modes is correlated reasonably well with the pattern of standard supergranulation detected using different techniques. However only about $66 \%$ of total energy is confined in the first four modes (excluding the solar rotation), the remaining fraction being spread among nearby modes. Then, even if the first four modes suffice for reproducing both the supergranulation pattern and the "5-min" oscillations, this is a strong indication that the modes interact with one an other and cannot be completely separated.

2. We were able to reproduce the spatial pattern $\Psi_{j}(x, y)$ that contributes to the velocity field corresponding to each eigenmode. Then we reproduced not only the supergranular pattern, but also the spatial pattern associated to the $p$-modes.

What we found must be considered as the result of a technique that can be successfully applied to photospheric motions to gain some insight into the physical behavior of supergranular and oscillatory motions, as well as their interactions. Indeed, little is known about the interaction of solar oscillations and convection in the solar atmosphere (Stix 1992). Convection might be a source of damping for oscillations (Zhevakin 1953), but it should be seen also as the main contribution for a stochastic excitation of the oscillatory behavior (Lighthill 1952; Goldreich \& Keeley 1977). Our results can be used as a key to improving knowledge in this direction. Of course we need longer time series in order to better investigate of characteristic frequencies and in order to investigate the role played by super-convective cells. The POD applied to photospheric velocity maps could be a useful tool for improving ground-based studies of the spatiotemporal evolution of supergranulation, thus reducing the 

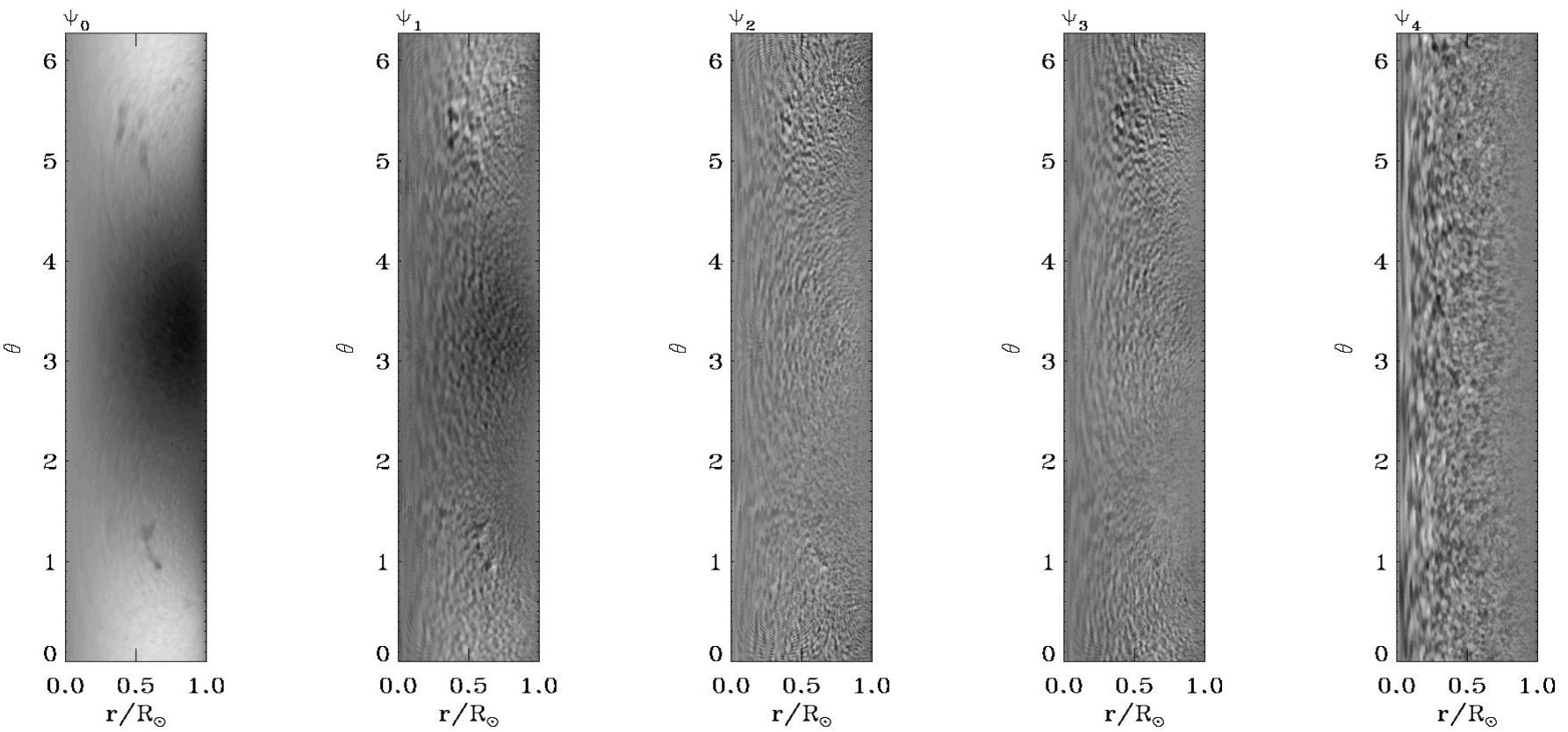

Fig. 6. The first five POD eigenfunction represented in the $(r, \theta)$ plane.
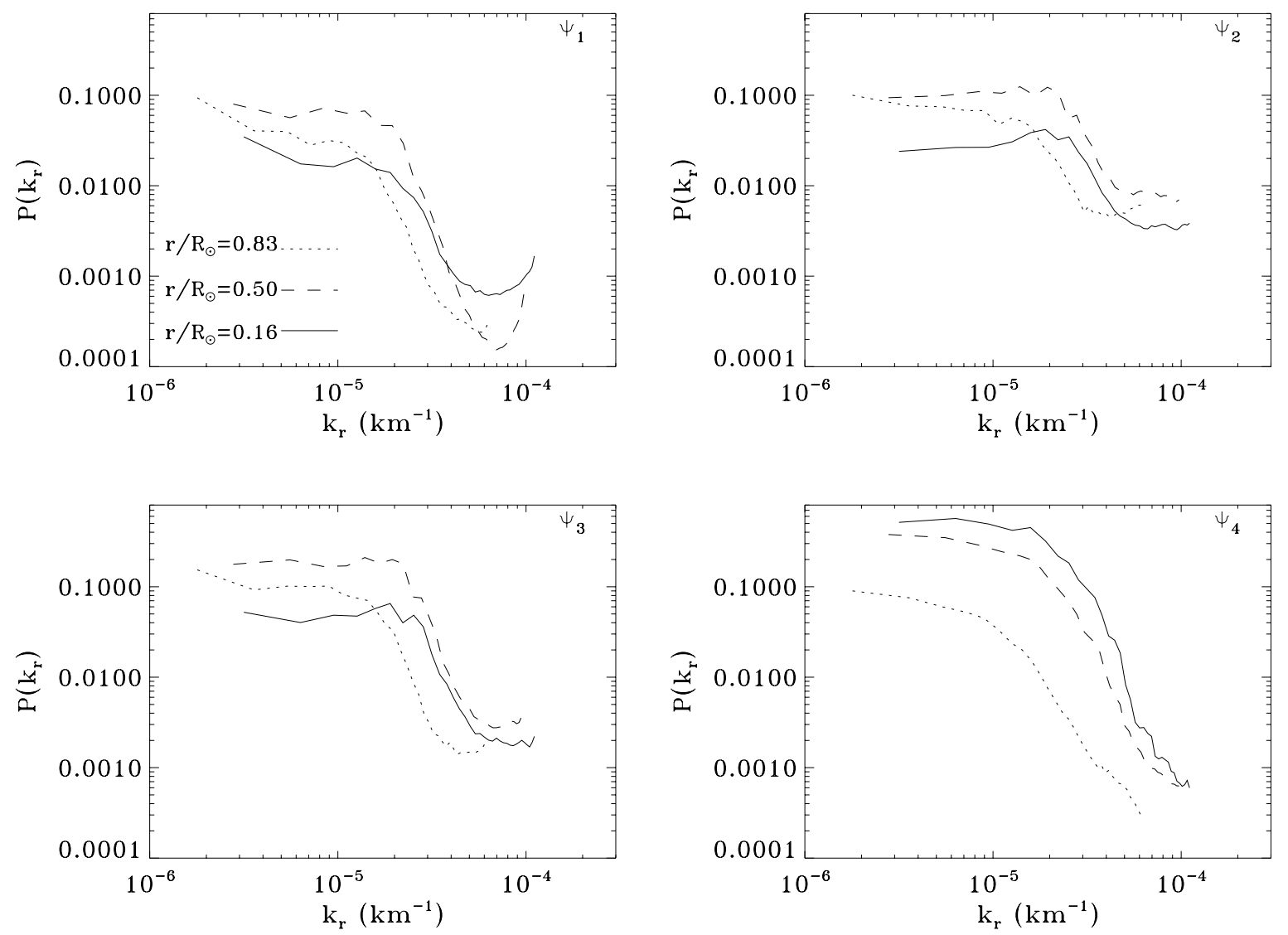

Fig. 7. Radial wavevector spectra for the eigenfunction $\Psi_{1}, \Psi_{2}, \Psi_{3}, \Psi_{4}$ calculated for the three different radial bands.

problem of the short data set duration. The POD, applied to longer photospheric velocity time series, gives us the possibility of isolating the rotation contribution in the most energetic mode. The results of the POD applied to high resolution data set are discussed in Vecchio et al. (2005).
Acknowledgements. I am grateful to the MOF Group and, in particular to P. F. Moretti, for providing the observations used in this work A particular acknowledgement goes to V. Carbone, F. Lepreti, L. Sorriso-Valvo, L. Primavera, N. Décamp, and P. Veltri for useful discussions. 


\section{References}

Alfonsi, G., \& Primavera, L. 2002, J. Flow visualization and Image Processing, 9, 89

Bray, R. J., Loughhead, R. E., \& Durrant, C. J. 1984, The Solar Granulation, 2nd edn. (Cambridge: Cambridge Univ. Press)

Carbone, V., Lepreti, F., Primavera, L., et al. 2002, A\&A, 381, 265

Cristensen-Dalsgaard, J. 2002, Rev. Mod. Phys., 74, 1073

De Rosa, M., Duvall, T. L., Jr., \& Toomre, J. 2000, Sol. Phys., 192, 351

Deubner, F.-L. 1971, Sol. Phys., 17,6

Giovannelli, R. G. 1980, Sol. Phys., 67, 211

Goldreich, P., \& Keeley, D. A. 1977, ApJ, 212, 243

Hart, A. B. 1954, MNRAS, 114, 17

Hart, A. B. 1954, MNRAS, 116, 38

Hathaway, D. H., Beck, J. G., Han, S., \& Raymond, J. 2002, Sol. Phys., 205, 25

Holmes, P., Lumley, J. L., \& Berkooz, G. 1996, Turbulence, Coherent Structures, Dynamical Systems and Symmetry (Cambridge Univ. Press)

Küveler, G. 1983, Sol. Phys., 88, 13

Leighton, R. B., Noyes, R. W., \& Simon, G. W. 1962, ApJ, 135, 474

Lighthill, M. J. 1952, Proc. R. Soc. London A, 211, 564

Lisle, J., De Rosa, M., \& Toomre, J. 2000, Sol. Phys., 197, 21

Mininni, P. D., Gòmez, D. O., \& Mindlin, G. B. 2002, Phys. Rev. Lett., 89,061101
Moretti, P. F., \& MOF Development Group, 200, Sol. Phys., 194, 1

Muller, R., Auffret, H., Roudier, Th., et al. 1992, Nature, 356, 322

November, L. J. 1994, Sol. Phys., 154, 1

November, L. J., \& Simon, G. W. 1988, ApJ, 333, 427

Rimmele, T., \& Schröter, E. H. 1989, A\&A, 221, 137

Roudier, Th., Rieutord, M., Malherbe, J. M., \& Vigneau, J. 1999, A\&A, 349, 301

Simon, G. W. 1967, ZAp, 65, 345

Simon, G. W., \& Leighton, R. B. 1964, ApJ, 140, 1120

Simon, G. W., Title, A. M., Topka, K. P., et al. (the SOUP team) 1988, ApJ, 327, 964

Shine, R. A., Simon, G. W., \& Hurlburt, N. E. 2000, Sol. Phys., 193, 313

Spruit, H. C., Nordlund, Å, \& Title, A. M. 1990, ARA\&A, 28, 236 S

Srikanth, R., Singh, J., \& Raju, K. P. 2000, ApJ, 534, 1008

Stix, M. 1991, The Sun: An Introduction (Springer Verlag)

Ulrich, R. K. 1970, ApJ, 162, 993

Vecchio, A., Carbone, V., Lepreti, F., et al. 2005, Phys. Rev. Lett., 95, 011102

Wang, H., \& Zirin, H. 1988, Sol. Phys., 115, 205

Wang, H., Tang, F., Zirin, H., \& Wang, J. 1996, Sol. Phys., 165, 223

Zhang, J., Wang, J., Wang, H., \& Zirin, H. 1998, A\&A, 335, 341

Zhevakin, S. A. 1953, Russian Astron. J., 30, 161 